\title{
Bacteriological Spectrum of Different Infections and their Antibiogram at NICVD, Dhaka
}

\author{
MS Shahidullah ${ }^{1}$, MA Yusuf ${ }^{1}$, Z Khatun $^{1}$, UKMN Ara $^{2}$, M Aziz $^{3}$, MR Rahman $^{3}$, F Hafiz $^{4}$ \\ ${ }^{1}$ Department of Microbiology, NICVD, ${ }^{2}$ Department of Paediatrics, Dhaka Medical College \\ Hospital, ${ }^{3}$ Department of Cardiology, NICVD, ${ }^{4}$ Department of Physical medicine, NICVD
}

Key Words:

Antibiogram, antibiotic

sensitivity pattern, aerobic bacteria.

\begin{abstract}
Background: One of the major causes of death in the current era is the infectious diseases. Aerobic bacteria are one of the most commonly isolated organisms from hospitalised patients. Objectives: The aim of the present study was to observe the infections caused by aerobic bacteria and their antibiotics susceptibility pattern.
\end{abstract}

Methods: This retrospective study was carried out in the National Institute of Cardiovascular Diseases (NICVD) from January 2012 to December 2012 for a period of one year. Patients who were admitted in medical wards and medical ICU suffering from different infections were undertaken for this study. Proper thoroughly clinical examination, routine and specific investigations were done in each case. Microbiological samplings were tried on day 1, after completion of antibiotic therapy or in between as required. Aerobic bacterial culture and sensitivity tests were done according to clinical laboratory standard institute (CLSI) standard.

Result: A total of 660 samples were studied of which male (70.0\%) were predominant than female (30.0\%). The highest number of patients was in the age group of 30-60 years (54.0\%) followed by 15-30 years (21.5\%) and less than 15 years (13.0\%). The mean age with standard deviation was $38.61 \pm 19.236$ years. The most common isolated bacteria was Escherichia coli (40.1\%) followed by Pseudomonas species (30.4\%), coagulase negative Staphylococcus (19.0\%) and coagulase positive Staphylococcus (5.9\%); however, beta-haemolytic Streptococcus (4.2\%) was detected. Urine culture has yielded Pseudomonas species (13.3\%), E. coli (71.1\%) and CNS (15.0\%). From pus Pseudomonas species (37.3\%) was isolated mostly which was 62 cases followed by E. coli (31.3\%), CNS (19.3\%) and CPS (7.2\%). Pseudomonas species was resistant to penicillin, amoxycillin and vancomycin and $\sim 50 \%$ resistant to cotrimoxazole, cefuroxim, ceftriaxone, piperacillin, azythromycin, cephalexin, netelmycin and pfloxacillin.

Conclusion: In the conclusion, majority bacteria are resistant to commonly used antibiotics.

(Cardiovasc. j. 2014; 6(2): 127-132)

\section{Introduction:}

Aerobic bacteria isolated from different clinical specimens have developed resistance against the major classes of antibiotics. ${ }^{1}$ Many of these resistance mechanisms are widespread among common pathogens and cause considerable concern in several clinical situations in which treatment options have become very limited. Cardiovascular intervention is very critical to handle. A great cardiovascular surgery by an expert surgeon can go in vein due to the infection and will go more serious if that infection is caused by resistant one. It is a great concern that extended-spectrum â-lactamase-producing Escherichia coli and Klebsiella pneumoniae are increasingly reported ${ }^{2-4}$ and have been associated with high mortality in adults. ${ }^{5}$ These
ESBL are frequently resistant to broad-spectrum cephalosporins. ${ }^{1}$ Similarly, the incidence of methicillin-resistant Staphylococcus aureus (MRSA) and vancomycin-resistant Enterococcus faecium has increased noticeably. ${ }^{6}$ Even among new antibiotic classes like oxazolidinones and the ketolides, there are reports of resistant bacteria. $^{1}$

The emerging resistance to the antibiotic creates problem during the treatment of infections among the cardiovascular disease patients. ${ }^{7}$ Thus, there is always a demand of surveillance to monitor current resistance pattern of the commonly used antibiotics. The purpose of the present study was to see the current levels of antibiotic resistance to predominant pathogens encountered in different infections at a tertiary care hospital.

Address of Correspondence : Dr. Md. Md. Sk Shahidullah, Department of Microbiology, National Institute of Cardiovascular Diseases, Dhaka, Bangladesh. Email: shahiddr1973@yahoo.com. 


\section{Methodology:}

This was a retrospective study conducted in the Department of Microbiology at National Institute of Cardiovascular Disease (NICVD), Dhaka to find out the aerobic bacterial agents causing different infections as well as their antibiotic sensitivity pattern. NICVD is the referral tertiary care hospital for cardiovascular diseases in Bangladesh. All patients at any age with both sexes presented with infections admitted at NICVD from July 2012 to June 2013 for a period of $01(\mathrm{One})$ year were enrolled for this study. Specimens were collected according to the type of infections. The most common specimen were blood, urine, pus, pericardial fluid, swab from wound, conjunctiva and throat. All these samples were received for microbiological examination. The specimens were inoculated into blood agar media, chocholate agar media, MacConkey's agar media and nutrient agar media. Bacterial species were identified by standard procedures. ${ }^{8,9}$ Antibiotic sensitivity test was performed by disc diffusion method (Kirby-Bauer's technique) using commercially available antibiotic discs (HiMedia, India) according to appropriate antibiotics panel to a specific bacterial strain. The antibiotic discs were applied manually into the surface of the inoculated agar plate and were incubated into an aerobic incubator (Memmert Company Ltd., Germany) for $18 \mathrm{~h}$ at $37^{\circ} \mathrm{C}$. The zones of inhibition for each antibiotic were measured in millimeters by measuring scale and were compared with values provided by the Clinical and Laboratory Standards Institute. ${ }^{10}$ The commonly used antibiotic disks were amoxicillin, amikacin $(30 \mathrm{mcg})$, ciprofloxacin $(5 \mathrm{mcg})$, cotrimoxazole $(1.25 / 23.75 \mathrm{mcg})$, ceftriaxone (30 $\mathrm{mcg})$, cephalexin (30 mcg), ceftazidime (10 mcg), gentamycin $(10 \mathrm{mcg})$, tobramycin $(10 \mathrm{mcg})$, imipenem, netilmycin, vamcomycin $(5 \mathrm{mcg})$. Statistical analysis was performed by SPSS 19.0 (Statistical Package for Social Science, Chicago, USA).

\section{Results:}

A total of 660 cases were studied of which male were predominant than female which were $462(70.0 \%)$ cases and $198(30.0 \%)$ cases respectively. The highest number of patients was in the age group of 30-60 years which was $357(54.0 \%)$ cases followed by $15-30$ years and less than 15 years which were $142(21.5 \%)$ cases and $86(13.0 \%)$ cases respectively. The mean age with standard deviation was $38.61 \pm 19.236$ years (range 1-90 years) (Table I).
Table-I

Distribution of Study Population According to Age and Sex $(n=660)$

\begin{tabular}{|c|c|c|c|}
\hline \multicolumn{3}{|c|}{ Age Group } & \multirow[t]{2}{*}{ Total } \\
\hline & Male & Female & \\
\hline$<15$ & $65(14.1 \%)$ & $21(10.6 \%)$ & $86(13.0 \%)$ \\
\hline $15-30$ & $88(19.0 \%)$ & $54(27.3 \%)$ & $142(21.5 \%)$ \\
\hline $30-45$ & 129(27.9\%) & $66(33.3 \%)$ & $195(29.5 \%)$ \\
\hline $45-60$ & $120(26.0 \%)$ & $42(21.2 \%)$ & $162(24.5 \%)$ \\
\hline$>60$ & $60(13.0 \%)$ & $15(7.6 \%)$ & $75(11.4 \%)$ \\
\hline Total & $462(100.0 \%)$ & $198(100.0 \%)$ & $660(100.0 \%)$ \\
\hline
\end{tabular}

* Pearson Chi-Square has been done to see the association; $p$ value $=0.018$

Mean age $\pm \mathrm{SD}=38.61 \pm 19.236$ (Range: 1 to 90 years)

Among 660 clinical specimens the growth of bacteria was in 289(43.8\%) cases and the rest $371(56.2 \%)$ specimens were yielded no growth (Table II).

Table-II

Rate of Bacterial Growth from Different Specimens $(n=660)$

\begin{tabular}{lcc}
\hline Culture & Frequency & Percentage \\
\hline Positive & 289 & 43.8 \\
Negative & 371 & 56.2 \\
\hline Total & 660 & 100.0 \\
\hline
\end{tabular}

The most common isolated bacteria was Escherichia coli which was 116(40.1\%) followed by Pseudomonas species, coagulase negative Staphylococcus and coagulase positive Staphylococcus which were 88(30.4\%), 55(19.0\%) and $17(5.9 \%)$ respectively; however, betahaemolytic Streptococcus was detected in $12(4.2 \%)$ cases (Table III).

Table-III

Distribution of Different Isolated Bacteria from Positive Culture $(n=289)$

\begin{tabular}{lcc}
\hline Isolated Bacteria & Frequency & Percentage \\
\hline Escherichia coli & 116 & 40.1 \\
Pseudomonas species & 88 & 30.4 \\
CNS & 55 & 19.0 \\
CPS & 17 & 5.9 \\
Streptococcus pyogenes & 12 & 4.2 \\
Proteus species & 01 & 0.4 \\
\hline Total & 289 & 100.0
\end{tabular}

*Coagulase positive Staphylococcus $=$ CPS

* Coagulase Negative Staphylococcus= CNS 
Most common specimen was urine 276(41.8\%) which had been given only 60(21.7\%) positive growth. Pus, wound swab, blood and throat swab which were $229(34.7 \%)$ cases, $73(11.1 \%)$ cases, $36(5.5 \%)$ and $21(3.2 \%)$ cases respectively were yielded 166(72.5\%), 43(58.9\%), 3(8.3\%) and $10(47.6 \%)$ cases positive growth. Culture of urine has yielded Pseudomonas species, E. coli and CNS which were $8(13.3 \%), 43(71.1 \%)$ and 9(15.0\%) respectively. From pus Pseudomonas species was isolated mostly which was 62(37.3\%) cases followed by E. coli, CNS and CPS which were $52(31.3 \%), 32(19.3 \%)$ and $12(7.2 \%)$ respectively. One $E$. coli and two Pseudomonas species were isolated from blood. In wound swab the most common isolated bacteria was Pseudomonas species, E. coli and CNS which were $12(27.9 \%), 11(25.6 \%)$ and 11(25.6\%) (Table III).

Pseudomonas species was mostly resistant to penicillin, amoxycillin and vancomycin and $\sim 50 \%$ resistant to cotrimoxazole, cefuroxim, ceftriaxone, piperacillin, azythromycin, cephalexin, netelmycin and pfloxacillin. It was found that Pseudomonas species was still more than $90.0 \%$ sensitive only to imipenem. Escherichia coli was more than $80.0 \%$ sensitive to only imipenem and amikacin. Coagulase negative Staphylococcus (CNS) was sensitive in imipenem, novobiocin and netelmycin. Coagulase positive Staphylococcus (CPS) was sensitive to only imipenem and cephalexin (Table V).

\section{Table-IV}

Distribution of most frequently received specimens according to Positive Bacterial Growth (n=289)

\begin{tabular}{lcccccc}
\hline Isolated Bacteria & Pus & Urine & Blood & Wound SwabThroat Swab & Others \\
\hline Pseudomonas species & $62(37.3 \%)$ & $8(13.3 \%)$ & $1(33.3 \%)$ & $12(27.9 \%)$ & $3(30.0 \%)$ & $2(28.6 \%)$ \\
E. coli & $52(31.3 \%)$ & $43(71.1 \%)$ & $2(66.7 \%)$ & $11(25.6 \%)$ & $3(30.0 \%)$ & $5(71.4 \%)$ \\
CNS & $32(19.3 \%)$ & $9(15.0 \%)$ & $0(.0 \%)$ & $11(25.6 \%)$ & $3(30.0 \%)$ & $0(0.0 \%)$ \\
CPS & $12(7.2 \%)$ & $0(0.0 \%)$ & $0(.0 \%)$ & $5(11.6 \%)$ & $0(0.0 \%)$ & $0(0.0 \%)$ \\
Streptococcus pyogenes & $7(4.2 \%)$ & $0(0.0 \%)$ & $0(.0 \%)$ & $4(9.3 \%)$ & $1(10.0 \%)$ & $0(0.0 \%)$ \\
Proteus species & $1(0.6 \%)$ & $0(0.0 \%)$ & $0(0.0 \%)$ & $0(0.0 \%)$ & $0(0.0 \%)$ & $0(0.0 \%)$ \\
\hline Total & $166(100.0 \%)$ & $60(100.0 \%)$ & $3(100.0 \%)$ & $43(100.0 \%)$ & $10(100.0 \%)$ & $7(100.0 \%)$ \\
\hline
\end{tabular}

* Pearson Chi-Square has been done to see the association which is corrected by Fisher's Exact Test; p value (95\% CI) = 0.026 (0.024-0.027); others= Pericardial fluid, endotracheal tube, sputum, stool, serous fluid; Coagulase positive Staphylococcus= CPS; Coagulase Negative Staphylococcus $=$ CNS; E. coli=Escherichia coli

\section{Table-V(a)}

Distribution of isolated bacteria with Resistant Pattern

\begin{tabular}{lccccccccc}
\hline Bacteria name & Tobra & Genta & Ceftazi & Cotrimo & Cipro & Imipe & Cefuro & Ceftri & Amika \\
\hline Pseudomonas & 2.4 & 28.2 & 35.3 & 46.2 & 36.5 & 7.1 & 55.3 & 50.6 & 15.3 \\
E coli & - & 48.5 & 68.8 & 50.0 & 49.1 & 6.1 & 34.2 & 54.4 & 15.3 \\
CNS & - & 21.8 & 40.0 & 1.8 & 56.4 & 3.6 & 30.9 & 30.9 & 12.7 \\
CPS & - & 20.0 & 50.0 & 00 & 52.9 & 5.9 & 41.2 & 58.8 & 17.6 \\
Strep pyogenes & - & 41.7 & 66.7 & - & 41.7 & - & 16.7 & 58.3 & 25.0 \\
Proteus species & - & 100.0 & 100.0 & - & - & - & - & - & - \\
\hline
\end{tabular}

*Tobra $=$ tobramycin, genta $=$ gentamycin, ceftazi $=$ ceftazidime, cipro $=$ ciprofloxacin, imipe $=$ imipenem, cefuro $=$ cefuroxim, ceftri= ceftriaxone, amika= Amikacin, Staph aureus= Staphylococcus aureus, Staph saprop= Staph saprophyticus, E. coli= Escherichia coli 
Table-V(b)

Distribution of isolated bacteria with sensitivity pattern

\begin{tabular}{lccccccccc}
\hline Bacteria name & Piper & Penicil & Azyth & Amoxy & Cephale & Clarith Vancom & Netel Pfloxaci \\
\hline Pseudomonas & 55.3 & 8.2 & 30.6 & 29.4 & 41.2 & - & 7.1 & 9.4 & 47.1 \\
E coli & 47.9 & 13.2 & 32.5 & 26.3 & 45.6 & - & 1.8 & 7.0 & 50.0 \\
CNS & 58.2 & 10.9 & 52.7 & 14.5 & 20.0 & 3.6 & 12.7 & 5.5 & 58.2 \\
CPS & 64.7 & - & 29.2 & 17.6 & 52.9 & - & 11.8 & 11.8 & 58.8 \\
Strep pyogenes & 58.3 & 8.3 & 58.3 & - & 16.7 & - & 8.3 & 16.7 & 33.3 \\
Proteus species & 100.0 & - & - & 100.0 & - & - & - & - & 100.0 \\
\hline
\end{tabular}

*Piper= Piperacillin, penicil= penicillin, azyth= azithromycin, cephale $=$ cephalexin, clarith $=$ clarithromycin, vancom $=$ Vancomycin, novobio $=$ novobiocin, netel $=$ netelmycin, pfloxaci $=$ pfloxacin, Staph aureus $=$ Staphylococcus aureus, Staph saprop $=$ Staph saprophyticus, E. coli= Escherichia coli

\section{Discussion:}

The antibiotic sensitivity pattern of organisms is changing very rapidly over a short period. ${ }^{11}$ It is particularly true for developing countries like Bangladesh where antibiotics are prescribed irrationally not only by the medical practitioners but the antibiotics are also purchased directly from the chemists like medicine shop keepers without prescription. ${ }^{7}$ Palikhe reported similar reason. ${ }^{12}$ It has been advised that clinicians should be aware of the rising resistance of bacteria to commonly prescribed antibiotics as well as the profile of antibiotic resistance. ${ }^{13}$ Therefore, for rational and appropriate use of antibiotics periodic evaluation of sensitivity pattern is essential. ${ }^{14,15}$

Male (70.0\%) was predominant than female (30.0\%) in this study. The specimen was collected from the cardiac referral tertiary care hospital in Bangladesh and regarding this cardiovascular diseases majority of the patients was male. That's why the findings of this study are correlated with this scenario. It is seen that the highest number of patients were in the age group of 30-60 years (54.1\%) followed by $10-30$ years $(21.5 \%)$ and more than 60 years $(11.4 \%)$. The mean age with standard deviation was $38.61 \pm 19.236$ years (range 1-90 years). The overall growth positive rate $(37.2 \%)$ in this study was in agreement with previous studies in Nepal. ${ }^{11,12}$ Escherichia coli (40.1\%) was the most common isolated bacteria followed by Pseudomonas species (30.4\%), CNS (19.0\%) and CPS (5.9\%). It is also found that Pseudomonas species (37.3\%), CNS (13.0\%) and CPS (4.3\%) were both found most commonly in pus. These findings agree with those reported by Anguzu and Olila on different infections where the most common wound contaminant was Pseudomonas species and Staphylococcus aureus. ${ }^{16}$ The findings also agree with those of Buwembo who identified Staphylococcus aureus as the commonest causative agent of potentially contaminated wounds. ${ }^{17}$ Nasal carriage of $S$. aureus is an important risk factor for infection of surgical site as the organism is a normal flora in the nostrils. ${ }^{7}$ Again, it is found that with the disruption of natural skin barrier Staphylococcus aureus, which is a common bacterium on surfaces of the body, can easily find the way into breach of the skin surfaces. The high prevalence of Pseudomonas infection may be because it is an exogenous source of infection and it is assumed that infection with this organism may also be due to contamination from the hospital environment. ${ }^{12}$

It is found that $56.2 \%$ specimen had no bacterial growth. This could be due to prior use of antibiotic, or may be the normal healing process where the bacteria have been overpowered by body's defence mechanism, antimicrobial activity in patients circulation since all of them had been on antibiotic therapy post operatively at time of collecting the samples or adequate nursing care like use of antiseptics for cleaning the wounds. ${ }^{12}$ It is also possible that some organisms could have been anaerobic bacteria that were missed as cultures were incubated aerobically. This condition could not therefore support growth of such organisms ${ }^{12}$. In this study the most 
common specimen was urine $(41.8 \%)$ followed by pus (34.7\%), blood (5.5\%) and wound swab (11.7\%). From all pus specimens $72.5 \%$ have shown positive growth. Urine samples have shown $21.7 \%$ positive growth. Interestingly majority (91.7\%) blood samples were shown negative growth. Reason of this may be due to prior intake of antibiotic leading to suppression of bacteria.

From pus specimen Pseudomonas species (37.3\%) was most commonly found and next to this is the E. coli (31.3\%). However, CNS and CPS were detected from pus in $19.3 \%$ and $7.2 \%$ respectively. Streptococcus pyogenes was isolated in $4.2 \%$ cases. Rahman et al has reported similar finding. ${ }^{18}$ Almost similar findings was reported by Shrestha et al in Nepal and observed that bacterial growth in $35.7 \%$ of the pus samples collected from all age groups. ${ }^{19}$ On the contrary, this was much higher than this present findings among adults as have been reported by Rajbhandari et al, ${ }^{20}$ Rai et al, ${ }^{11}$ Shrestha et al ${ }^{19}$ and Chhetri et al. ${ }^{21}$ This discrepancy could be because of the age of the subjects included in those studies. Another reason may be due to the sample size of the present study.

In this present study urine was yielded $E$. coli (71.1\%) as most common bacteria followed by CNS (15.0\%) and Pseudomonas species (13.3\%). Similar result was reported by Rahman et al ${ }^{18}$ and Rai et al. ${ }^{11}$ Pseudomonas species was maximum resistant to penicillin, amoxycillin and vancomycin; however, resistant to cotrimoxazole, cefuroxim, ceftriaxone, piperacillin, azythromycin, cephalexin, netelmycin and pfloxacillin was also significant. However Pseudomonas species are still sensitive only to imipenem. The resistance observed in Pseudomonas species could also be attributed to irrational use of antibiotics for conditions that may not clinically indicate their use, over-thecounter sell of antibiotics in pharmacies without prescription by authorised practitioners, some new drug formulations which may be of poor quality and dumping of banned products into the market where the public may get access to them. ${ }^{12}$ In view of the resistance observed, infections caused by MRSA can be expensive in terms of costs of treatment, morbidity and prolonged hospitalisation. ${ }^{18}$
Escherichia coli was sensitive to only imipenem and Amikacin. Similar result was reported by Rahman et $\mathrm{al}^{18}$ that most of the antibiotics were resistant to $E$ coli and mentioned that only ceftriaxone and ceftazidime were maximum sensitive. Staphylococcus saprophyticus was sensitive in imipenem, novobiocin and netelmycin. Staphylococcus aureus was sensitive to only imipenem and cephalexin. Similar sensitivity pattern was reported by Sharma, Kumari et al and Bhandari. ${ }^{22-24}$ The resistance shown to amoxycillin and ampicillin may be due to the antibiotics having been in use for much longer time and their oral route of administration that affects their rate of absorption into blood stream. ${ }^{12}$ Some of them were used as prophylaxis therefore increasing their use in patients. Over-use of antibiotics contributes to organisms developing resistance. ${ }^{25}$ In another study in Bangladesh Shamsuzzaman et al has reported that there is a trend of antibiotic resistant among the Pseudomonas species, E coli and Staphylococcus aureus isolated from different samples and has shown that the resistant pattern gradually increases among the isolated bacteria from different clinical specimens. ${ }^{26}$ This clearly indicates that antibiotic resistant is alarming to the community of this country. It is now an urgent need to develop antibiotic policy as soon as possible to save the future generation from these bugs.

\section{Conclusion:}

In the conclusion, majority bacteria are resistant to commonly used antibiotics. Since a high proportion of samples have positive cultures, infection control is recommended as a strategy to minimise spread of resistant organisms. Future studies should be extended to include cultures under anaerobic conditions to establish presence of other organisms that require such environment for growth. It is recommended that judicial antibiotic use should be carried out.

\section{References:}

1. Jones ME, Karlowsky JA, Draghi DC, Thornsberry C, Sahm DF, Bradley JS. Rates of antimicrobial resistance among common bacterial pathogens causing respiratory, blood, urine, and skin and soft tissue infections in paediatric patients. Eur J Clin Microbiol 2004;23(6):445-455.

2. Kim Y-K, Pai H, Lee H-J, et al. Bloodstream infections by extended-spectrum $\beta$-lactamase-producing Escherichia coli 
and Klebsiella pneumoniae in children: epidemiology and clinical outcome. Antimicrob Agents Ch 2002;46: 1481-1491.

3. Bush K, Jacoby G. Nomenclature of TEM $\beta$-lactamases. $J$ Antimicrob Chemoth 1997;39:1-3.

4. Jacoby GA, Medeiros AA. More extended-spectrum âlactamases. Antimicrob Agents Ch 1991;35: 1697-1704.

5. Paterson DL, Ko WC, Von Gottberg A, et al. Outcome of cephalosporin treatment for serious infections due to apparently susceptible organisms producing extendedspectrum â-lactamases: implications for the clinical microbiology laboratory. J Clin Microbiol 2001;39:22062212.

6. Sahm DF, Marsilio MK, Piazza G. Antimicrobial resistance in key bloodstream bacterial isolates: electronic surveillance with The Surveillance Network databaseUSA. Clin Infect Dis 1999;29:259-263.

7. Shahidullah MS, Yusuf MA, Khatun Z, Ara U, Mitul MT. Antibiotic Sensitivity Pattern of Bacterial Isolates from Different Clinical Specimens: Experience at NICVD, Dhaka. Cardiovasc J 2012;5(1):67-72.

8. Cheesbrough M. Medical Laboratory Manual for Tropical Countries. Cambridge University press, 2000;Vol. 11.

9. Lalitha MK. Manual on Antimicrobial Susceptibility Testing. $7^{\text {th }}$ edition. Delhi. Indian Association of Medical Microbiologists, 2004: 10-47.

10. Clinical and Laboratory Standards Institute (CLSI). Performance standards for antimicrobial susceptibility testing; Seventeenth informational supplement.M100-S17. 2010, CLSI, Wayne, PA

11. Rai GK, Upreti HC, Rai SK, Shah KP, Shrestha RM. Causative agents of urinary tract infections in children and their antibiotic sensitivity pattern: a hospital based study. Nepal Medical College Journal 2008; 10(2): 86-90.

12. Palikhe N. Prescribing pattern of antibiotics in pediatric hospital of Kathmandu Valley. Kathmandu Univ Med $J$ 2004;2: 6-12.

13. Oreskovic NM, Sembrano EU. Repeat urine cultures in children who are admitted with urinary tract infections. Pediatrics 2007: 119: 325-329.

14. Jones RN, Thornsberry C. Cefotaxime: a review of in vitro antimicrobial properties and spectrum of activity. Rev Infect Dis 1982; 4: 5300-5315.
15. Rimal HS, Sharma AK, Gami FC, Sharma PR. Urinary tract infections in febrile children without localizing signs. J Nepal Paediatr Soc 2006; 27: 31.

16. Anguzu JR, Olila D. Drug sensitivity patterns of bacterial isolates from septic post-operative wounds in a regional referral hospital in Uganda. African Health Sciences 2007; 7(1): 148-154.

17. Buwembo KBM. Post operative wound infection, Dissertation for M. Med Surgery, Makerere University, Kampala, 1990. 1-45

18. Rahman A, Rashid A, Begum SA, et al. Bacteriological aetiology of flood affected diarrhoeal patients admitted in Dhaka Medical College Hospital and antibiogram of the isolates. Bangladesh J Med Microbiol 2008;2(1):13-17.

19. Shrestha B, Basnet RB, Shrestha P, Shahi P. Prevalence of urinary tract infection in female patients attending Kathmandu Model Hospital. J Nepal Assoc Med Lab Sci 2005; 7: 10-14.

20. Rajbhandari R, Shrestha J. Bacteriological study of urinary tract infection and its antibiotic sensitivity test: a hospital based study. J Nepal Assoc Med Lab Sci 2002; 4: 26-32.

21. Chhetri PK, Rai SK, Pathak UN et al. Retrospective study of urinary tract infection at Nepal medical college teaching hospital, Kathmandu. Nepal Medical College Journal 2001; 3: $83-85$.

22. Hiramatsu K, Hanaki H, Ino T, Yabuta K, Oguri T, Tenover F.C. Methicillin-resistant Staphylococcus aureus clinical strain with reduced vancomycin susceptibility. $J$ Antimicrob Chemoth 1997; 40:135-136.

23. Sharma PR. Urinary infection: the infection that matters. J Inst Med (Nepal) 1983; 5: 19-22.

24. Kumari N, Ghimire G, Magar JK, Mahapatra TM, Rai A. Antibiogram pattern of isolates from UTI cases in Eastern part of Nepal. Nepal Medical College Journal 2005; 7 (2): 116-118.

25. Bhandari SD. Surgical Site Infection, Bacterial Isolates and Their Sensitivity Pattern at Surgical Ward. MJSBH 2012;11(2):18-20.

26. Shamsuzzaman AKM, Paul SK, Mahmud MC, Musa AKM, Hossain MA. Emerging antimicrobial resistant amongst common bacterial pathogens in Mymensingh medical College Hospital. Bangladesh J Med Microbiol 2007; 1(1):4-9. 